\title{
INDEPENDENT COMPONENT ANALYSIS AND DISCRETE WAVELET TRANSFORM FOR ARTIFACT REMOVAL IN BIOMEDICAL SIGNAL PROCESSING
}

\author{
Salvatore Calcagno, Fabio La Foresta and Mario Versaci \\ Department of Civil Engineering, Environment, Energy and Materials, \\ Mediterranea University of Reggio Calabria, Via Graziella Feo di Vito, I-89122 Reggio Calabria, Italy
}

Received 2012-10-04, Revised 2013-01-15; Accepted 2013-11-27

\begin{abstract}
Recent works have shown that artifact removal in biomedical signals can be performed by using Discrete Wavelet Transform (DWT) or Independent Component Analysis (ICA). It results often very difficult to remove some artifacts because they could be superimposed on the recordings and they could corrupt the signals in the frequency domain. The two conditions could compromise the performance of both DWT and ICA methods. In this study we show that if the two methods are jointly implemented, it is possible to improve the performances for the artifact rejection procedure. We discuss in detail the new method and we also show how this method provides advantages with respect to DWT of ICA procedure. Finally, we tested the new approach on real data.
\end{abstract}

Keywords: Artifact Removal, Discrete Wavelet Transform, Independent Component Analysis, Neural Networks, Surface EMG

\section{INTRODUCTION}

When the biomedical signals recordings are strongly corrupted by the artifacts, a preprocessing step is needed in order to extract some clinical information from the data (Mammone et al., 2012; Labate et al., 2011a; 2011b; Campolo et al., 2011; Inuso et al., 2007a). Artifact removal is a key topic in biomedical data interpretation and then it is powerful in various applications. For these reasons, in the last years many researchers have studied the artifact removal and they have shown that it is a critical problem in the biomedical signal processing. In particular, the researchers have observed how contamination of Electroencephalographic (EEG) activity by eye movements, blinks, cardiac signals and muscle and line noise remains today a serious problem for EEG interpretation and analysis, especially for some patient groups since rejecting contaminated EEG segments may result in an unacceptable data loss.
Parallelly, the authors worked to artifact modeling/removal in other fields of research maturing competences strongly helpful for the problem under study (Costantino et al., 2012; Cacciola et al., 2007; 2009; 2010; Angiulli and Versaci, 2002; 2003). Recently, some works have shown that a successful artifact removal procedure in biomedical signals, like Electromyographic (EMG) or EEG recordings, can be performed using Discrete Wavelet Transform (DWT) or Independent Component Analysis (ICA). In particular, authors have discussed the effectiveness of their method based on DWT in order to perform the cancellation of Stimulus Artifact (SA) in the serosal recordings of Gastric Myelectric Activity (GMA). In this case DWT method allows obtaining successfully artifact cancellation because the $\mathrm{SA}$ is a superimposed signal that is a combination of periodic rectangular pulses width of $0.3 \mathrm{sec}$ and frequency of $10 \%$ higher than the intrinsic gastric slow wave frequency.

Corresponding Author: Salvatore Calcagno, Department of Civil Engineering, Environment, Energy and Materials, Mediterranea University of Reggio Calabria, Via Graziella Feo di Vito, I-89122 Reggio Calabria, Italy 
This procedure fails when the artifacts do not have these properties, for example DWT approach cannot remove Electro Cardio Graphic (ECG) artifact present in some myoelectric recordings (Inuso et al., 2007b; La Foresta et al., 2005) because the spectral components of artifact overlap with the myoelectric signal spectrum. On the other hand, the ICA method, its implementations and its applications are well described in literature and a review on artifact identification and removal, with special emphasis on the ocular ones, can be also found. ICA was implemented in order to remove artifacts in EEG and Magneto Encephalogram (MEG) signals. Some problems can arise in some artifact removal techniques that may lead to the insertion of undesirable new artifacts into the brain recordings. Further methods are presented in literature. In these applications we need a considerable amount of data where the amplitude of the artifact is much higher than that of the EEG or MEG. Otherwise, it was shown that artifacts can indeed be estimated by using ICA alone. In fact, ICA is used with good results to remove the eye activity artifacts from visual EventRelated Potentials (ERP) in normal and clinical subjects. This approach is able to perform the artifact removal only for multichannel recordings, in which it is possible to isolate the artifact by means of statistical independence theory.

The objective of this study is to examine the applicability of DWT and ICA methods for removing artifacts from a surface Electromyography (sEMG). The use of DWT and ICA in biomedical signal processing was analyzed by many researchers, who have proposed several studies and books about this topic. The aim of this study is to discuss the use of these methods in order to perform artifact removal. Thus, we focus our attention on the performance of the various algorithms and we show their behaviors in different kinds of artifacts discussing the advantages and drawbacks of these techniques and analyzing applications in which DWT and ICA cannot remove some artifacts. Therefore, we show some cases in which the application of DWT or ICA algorithms does not provide good results. We also show that in these cases, a joint use of DWT-ICA allows improving the quality of results; in the following we refer to this method by the acronym WICA. We prove that ICA method has very good performance when the recordings are redundant, i.e., the observed data (mixtures) are equal or larger in number than independent sources; otherwise, classic ICA shows poor performances. This problem is often called ICA with over complete bases and it was solved by modified ICA methods. These methods are computationally rather inefficient and much more. Thus we propose to perform artifact cancellation by using a WICA method rather than modified ICA methods. We also compare the obtained results by the proposed methods. Finally we show a clinical application in which only the proposed method allows performing successfully artifact cancellation.

\section{STATEMENT OF THE PROBLEM: ARTIFACT REMOVAL METHODS}

The artifact is a signal that hides some useful information in a measured signal. It is noticeable to classify the artifacts according to their features both in time and in frequency domains. There are some artifacts that are well localized in frequency, whereas their influence is spread over all the time axis of the original signal. Others typologies of artifacts, instead, are confined to a small temporal area, while their spectral components cover almost all the frequency spectrum of the original signal. Only in a few described cases, a classic filtering is suitable. Thus advanced methods are generally needed to obtain good results. In the next subsections, we briefly discuss the DWT and ICA methods and their applications for artifact cancellation. We also present a method based on the joint use of DWT and ICA.

\subsection{DWT Method}

The wavelet analysis is a time-frequency representation introduced in order to overcome the limitations in time and in frequency resolution suffered by the classical Fourier techniques. The Wavelet Transform (WT) is a Multi-Resolution Analysis (MRA). Here, a scaling function $\mathrm{j}$ is used to create a series of approximations of a signal, each differing by a factor 2 (or by another fixed factor) from its nearest neighboring approximations. Additional functions y, called wavelets, are then used to encode the difference between adjacent approximations. In its discrete the wavelet transform is implemented by a bank of band-pass filters each having frequency band and central frequency half than the previous one. First the original signal $S(t)$ is passed through two filters, a low-pass and a high-pass one. An approximation signal, $\mathrm{A}(\mathrm{t})$, is extracted from the low pass filter, whereas from the high-pass signal a detail signal $\mathrm{D}(\mathrm{t})$ is taken out. In the standard tree of decomposition only the approximation signal is passed again through the second stage of filters and so again until the last level of the decomposition. For each level the frequency band of the signal and the sampling 
frequency are halved. The Wavelet Series Expansion (1) of a signal $x(t) \in L^{2}(R)$ is Equation (1):

$$
\mathrm{x}(\mathrm{t})=\sum_{\mathrm{k}} \mathrm{c} \varphi_{\mathrm{jbk}}\left(\mathrm{t}_{\mathrm{j}_{0} \mathrm{k}}\right)+\sum_{\mathrm{j}=\mathrm{j}_{0}}^{\infty} \sum_{\mathrm{k}} \mathrm{d} \psi_{\mathrm{jk}}\left(\mathrm{t}_{\mathrm{jk}}\right)
$$

Where:

$$
\mathrm{d}_{\mathrm{jk}}=\int_{-\infty}^{\infty} \mathrm{x}(\mathrm{t}) \psi_{\mathrm{j}, \mathrm{k}}^{*}(\mathrm{t}) \mathrm{dt}
$$

are called the detail or wavelet coefficients and:

$$
\psi_{j, k}(t)=\frac{1}{\sqrt{2^{j}}} \psi\left(\frac{t-k 2^{j}}{2^{j}}\right)
$$

are the wavelet functions. The approximation or scaling coefficients are:

$$
\mathrm{c}_{\mathrm{jk}}=\int_{-\infty}^{\infty} \mathrm{x}(\mathrm{t}) \varphi_{\mathrm{j}, \mathrm{k}}{ }^{*}(\mathrm{t}) \mathrm{dt}
$$

Where:

$$
\phi_{j, k}(t)=\frac{1}{\sqrt{2^{j}}} \phi\left(\frac{t-k 2^{j}}{2^{j}}\right)
$$

are the scaling functions the details and the approximations are defined in Equation (2):

$D_{j}(t)=\sum_{k=-=}^{+=} d_{j k} \cdot \psi_{j k}(t) A_{j}(t)=\sum_{k=-=}^{+=} c_{j k} \cdot \varphi_{j k}(t)$

and the final reconstruction of the original signal computed by the details and the approximations is described by Equation (3), for fixed $\mathrm{N}$ :

$\mathrm{S}(\mathrm{t})=\mathrm{A}_{\mathrm{N}}(\mathrm{t})+\mathrm{D}_{1}(\mathrm{t})+\mathrm{D}_{2}(\mathrm{t})+\ldots+\mathrm{D}_{\mathrm{N}}(\mathrm{t})$

The Discrete Wavelet Transform (DWT) can be used in order to perform artifact removal (Inuso et al., 2007a). Its application is based on the spectral separation between the original signal and the artifact: a good removal is possible only if the artifact spectral content is well localized (compactly supported). Thus, the wavelet decomposition is able to remove the artifact by means of the denoising procedure applied to the single wavelet details $D_{1}(t), D_{2}(t) \ldots D_{N}(t)$. If we have multichannel recordings the artifact removal must be performed separately, channel by channel, applying the same algorithm for each channel recording.

\subsection{ICA Method}

The Independent Component Analysis is a method to find underlying factors or components from multivariate (multidimensional) statistical data. What distinguishes ICA from other methods is that it looks for components that are both statistically independent and non-gaussian. The ICA solves the Blind Source Separation (BSS) problem to recover $\mathrm{n}$ independent source signals. Consider $\mathrm{N}$ samples of the observed data vector $\mathrm{x}$, whose elements are the mixtures $\mathrm{x}_{1}, \mathrm{x}_{2}, \ldots, \mathrm{x}_{\mathrm{m}}$, modeled by Equation (4):

$$
\mathrm{x}=\mathrm{As}=\sum_{\mathrm{j}=1}^{\mathrm{n}} \mathrm{a}_{\mathrm{j}} \mathrm{s}_{\mathrm{j}}
$$

where, $\mathrm{A}$ is the unknown m-by-n mixing matrix with column vectors $a_{j}, j=1,2, \ldots \ldots, n$ and $s$ is an unknown n-dimensional source vector containing the source signals $\mathrm{s}_{1}, \mathrm{~s}_{2}, \ldots, \mathrm{s}_{\mathrm{n}}$, which are assumed to be statistically independent. In general, the dimensionality $\mathrm{m}$ of the vector $x$ and $a_{j}$ can be different from $n$. Usually it is assumed that there are at least as many mixtures as source $(m \geq n)$, the mixing matrix A has full rank and that at most one of source $s_{\mathrm{j}}$ is Gaussian. The BSS techniques do not use any training data and do not assume any a priori knowledge about parameters of mixing systems (i.e., no knowledge about the matrix A). The ICA resolves the BSS problem under the hypothesis that the sources are statistically independent each others. In particular it consists in estimating a n-by-m matrix $\mathrm{W}$ such that Equation (5):

$\mathrm{u}=\mathrm{Wx}=\sum_{\mathrm{j}=1}^{\mathrm{m}} \mathrm{w}_{\mathrm{j}} \mathrm{x}_{\mathrm{j}}$

Such a model (5) can be used in different situations, for example in multidimensional signal processing, where each sensor receives an unknown superimposition of unknown source signals at time instants $t=1,2, \ldots$, $\mathrm{N}$. In the last ten years many algorithms were proposed in order to perform ICA. We investigate, in particular, on the INFOMAX algorithm implemented algorithm, we used the switching extended rule Equation (6):

$$
\Delta \mathrm{W} \infty\left[\mathrm{I}-\mathrm{K} \tanh (\mathrm{u}) \mathrm{u}^{\mathrm{T}}-\mathrm{uu}^{\mathrm{T}}\right] \mathrm{W}
$$

where, $\mathrm{K}$ is a $\mathrm{n}$-dimensional diagonal matrix, which $\mathrm{k}_{\mathrm{i}}$ elements are: 


$$
\left\{\begin{array}{l}
\mathrm{k}_{\mathrm{i}}=1: \text { supergaussian } \\
\mathrm{k}_{\mathrm{i}}=-1: \text { subgaussian }
\end{array}\right.
$$

We choose, $k_{i}$ related to the sign of the $u_{i}$ kurtosis: $k_{i}$ $=\operatorname{sign}\left(\operatorname{durt}\left(\mu_{\mathrm{i}}\right)\right)$. ICA allows performing artifact cancellation. The procedure consists in the extraction of the Independent Components (ICs), to identify the ICs related to the artifacts; the cleaned signals reconstruction is obtained by setting to zero in (4) the columns, related to the artifacts sources, of the matrix A. ICA can often be simplified by means of the Principal Component Analysis (PCA) preprocessing lightening the computational charge and then reducing the computational time. PCA extracts from a signal mixing some uncorrelated sources: Uncorrelatedness is a lower property than independence, i.e., two signals can be uncorrelated without being independent; conversely, if two signals are independent, they are also uncorrelated. PCA can be also used to reduce the data dimensionality and/or to perform whitening process, to obtain some unit variance components. Unlike the wavelet analysis, ICA approach can be applied only to multichannel recordings.

\subsection{Wavelet-Independent Component Analysis}

The WICA method, described in Fig. 1, encompasses the properties of the DWT and ICA methods. The main idea of WICA is to examine the statistical independence of the recordings by means of ICA algorithm applied only to corrupted wavelet details (Azzerboni et al., 2002). In other words, the ICA algorithm works only on a fixed frequency range. In the next section we will show that this method improves the performances of ICA and it allows extracting successfully the artifact components with less distortion. Thus, we perform the wavelet decomposition at a fixed level for each channel. In this phase, a user interface is needed in order to choose the appropriate decomposition level and to select the details that concern the spectral range in which the artifact is localized. Next ICA minimizes a measure of statistical independence of new data set. We restrict the ICA application only on the frequency regions of interest, where the artifacts should be localized. A first advantage of this technique is avoiding that the signal part which is certainly artifact-free be corrupted by the source separation algorithm. The main steps of WICA approach are discussed as follows: (a) Wavelet decomposition. We apply DWT to every channel of the recordings. So we obtain signals that have non-overlapping spectra. (b) Identification and selection of corrupted details. We identify and select only the details that contain some artifact components. So, we organize the selected details in a new data set. In the selection step the user interface is needed. (c) ICA of selected details. We apply ICA algorithm to new data set. A preprocessing step, PCA and/or whitening, is performed in order to reduce the data dimensionality and to lighten the computational charge. Thus, we estimate the ICs and we remove the ones related to the artifacts. (d) Reconstruction of selected details after artifact removal. The selected details are cleaned by artifact components by means of the procedure described in ICA method. (e) Wavelet reconstruction. We perform the wavelet reconstruction by (3) using the non-selected details and the cleaned details after ICA step. Finally, we obtain the multichannel recordings in which the artifacts are removed.

\section{SIMULATIONS: ALGORITMS TESTING}

The simulations of the artifact removal by means of DWT, ICA and WICA methods are shown in this section. We test the performances on synthesized signals. Also the artifacts are synthesized in order to test the quality of the different approaches. In order to give a quantitative value to the goodness of the algorithm, we compare in both time-and frequency-domains the original signal (when the artifact is not yet added) and the reconstructed one after the artifact removal.

\subsection{Synthesized Signals}

We synthesized six myoelectric-like signals (surface EMG with of six electrodes). They are 12000 samples digital signals equivalent to $12 \mathrm{sec}$ (sampling frequency $1 \mathrm{kHz}$ ). We also synthesized three typologies of artifacts, an impulsive artifact, a similar ECG artifact and an impulsive-ECG artifact (Fig. 2).

The impulsive artifact is composed by a periodic high frequency burst. This kind of artifact can be viewed like a stimulus signal. Moreover, its spectrum is well localized and it is almost all separated by the signal spectrum.

The mean frequency of the artifact spectrum is higher than the maximum frequency present in the original signal. This is the most suitable kind of signal identifiable by the DWT. The ECG-like artifact is a signal that represents the cardiac activity. In sEMG recordings, this artifact is often present and it cannot be removed by DWT method, because its spectrum is overlapped to the EMG one. This is the most suitable kind of signal identifiable by the ICA algorithm. The last artifact, impulsive-ECG, is obtained by linear combination of the two previous typologies. Thus, we use these artifacts to corrupt the original sEMG recordings and we test the goodness of the methods. 


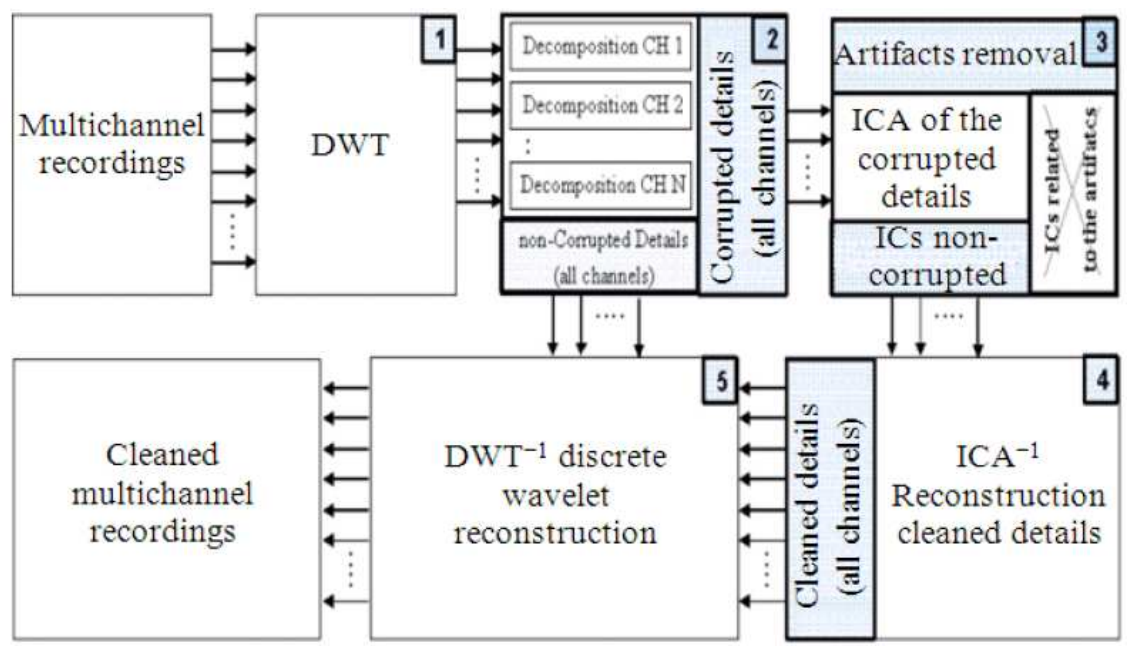

Fig. 1. Wavelet-ICA method. The procedure consists of five steps: (1) wavelet decomposition, (2) identification and selection of corrupted details, (3) ICA of selected details, (4) reconstruction of selected details after artifact removal and (5) wavelet reconstruction

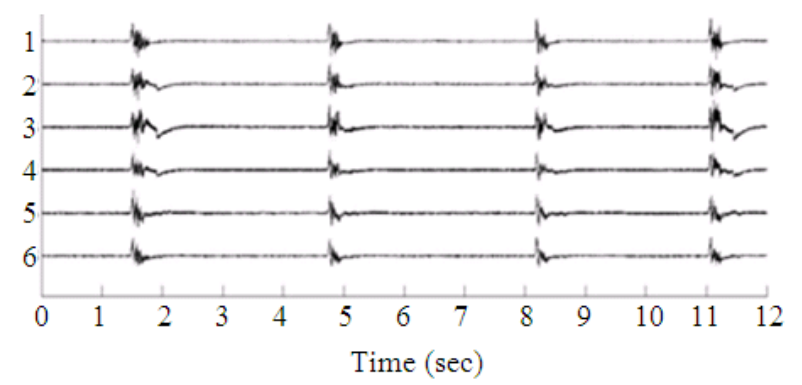

(a)

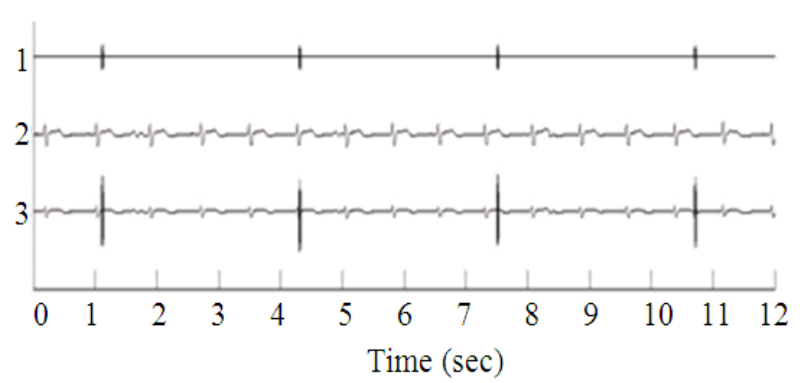

(b)

Fig. 2. (a) Synthesized six channels sEMG and (b) synthesized (1) impulsive artifact, (2) similar ECG artifact and (3) impulsive-ECG artifact

(a)

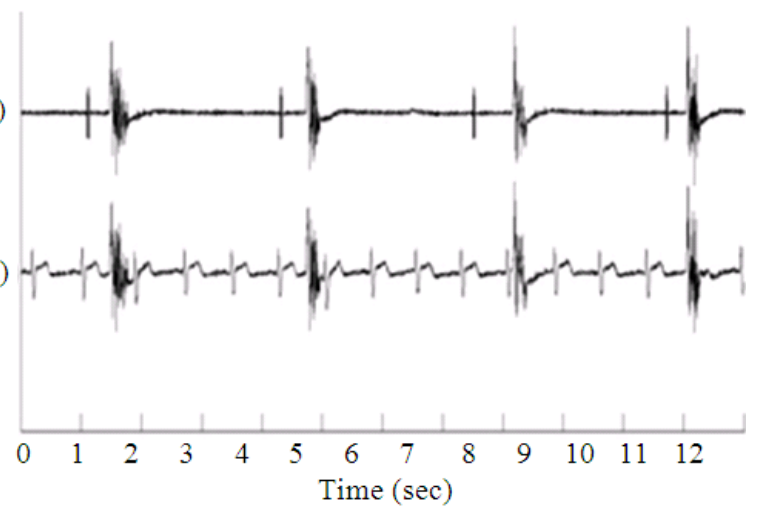

Fig. 3. The first channel of synthesized sEMG corrupted by (a) an impulsive artifact and (b) a similar ECG artifact

\subsection{Artifact Removal using DWT Method}

First, we corrupt the first channel of synthesized sEMG recordings with impulsive and similar ECG artifact, respectively. Figure $\mathbf{3}$ shows the corrupted signals. In Fig. 4 the six-level wavelet decomposition by DWT algorithm) is shown. This figure confirms the high ability of this method to separate the EMG signal by the impulsive artifact, which is almost all included in the first detail (that contains the highest frequencies). The kind of wavelet function used in this application is the fourth "Daubechies". On the other hand, we can see that the separation is bad for the EMG signal corrupted by similar ECG artifact. The original and reconstructed EMG signals are represented in Fig. 5. It is easy to see that the DWT method fails in the case of a corrupted ECG signal. Figure 5 also shows the goodness of the DWT method. 
Salvatore Calcagno et al. / American Journal of Applied Sciences 11 (1): 57-68, 2014

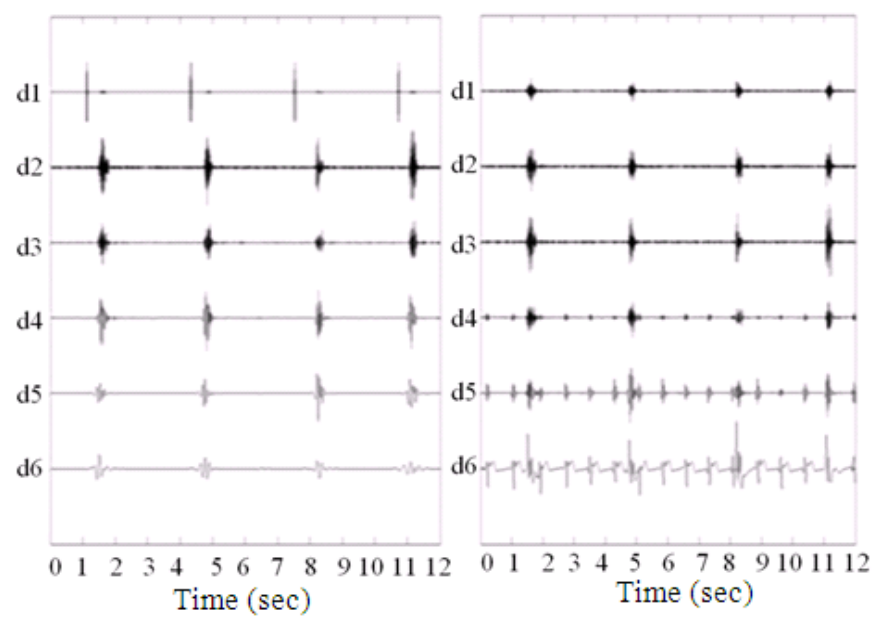

Fig. 4. The wavelet decomposition of corrupted channel by (left) an impulsive artifact and (right) a similar ECG artifact. In the first case the DWT allows to isolate the artifact contribution on the first detail, whereas in other case the DWT method can

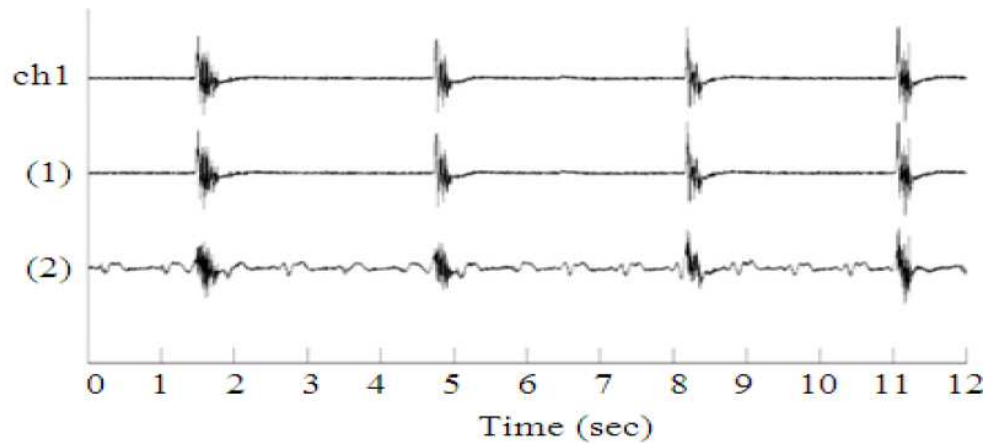

Fig. 5a. Performances of DWT method applied on the corrupted signals by the different artifact typologies shown in Fig. 3. Comparison after artifact removal in time domain: (ch 1) first channel of synthesized sEMG, (1) reconstructed signal after impulsive
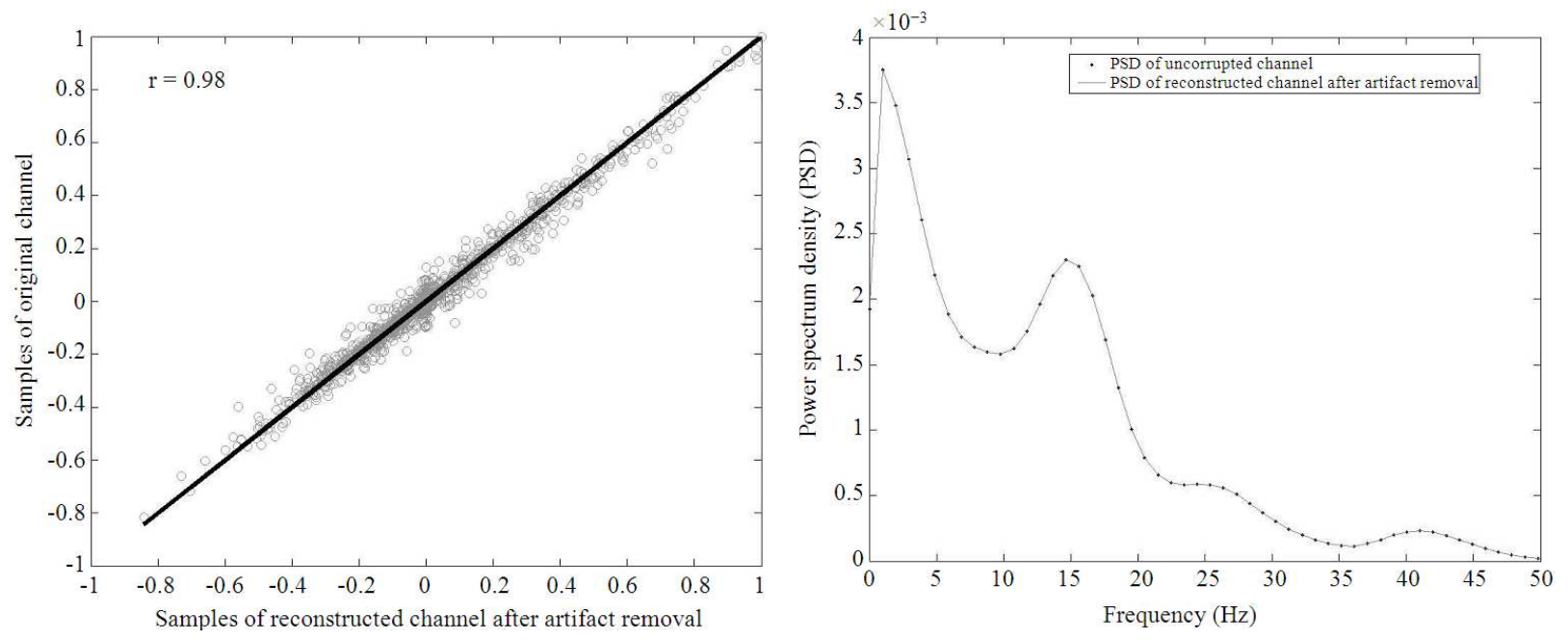

Science Publications 

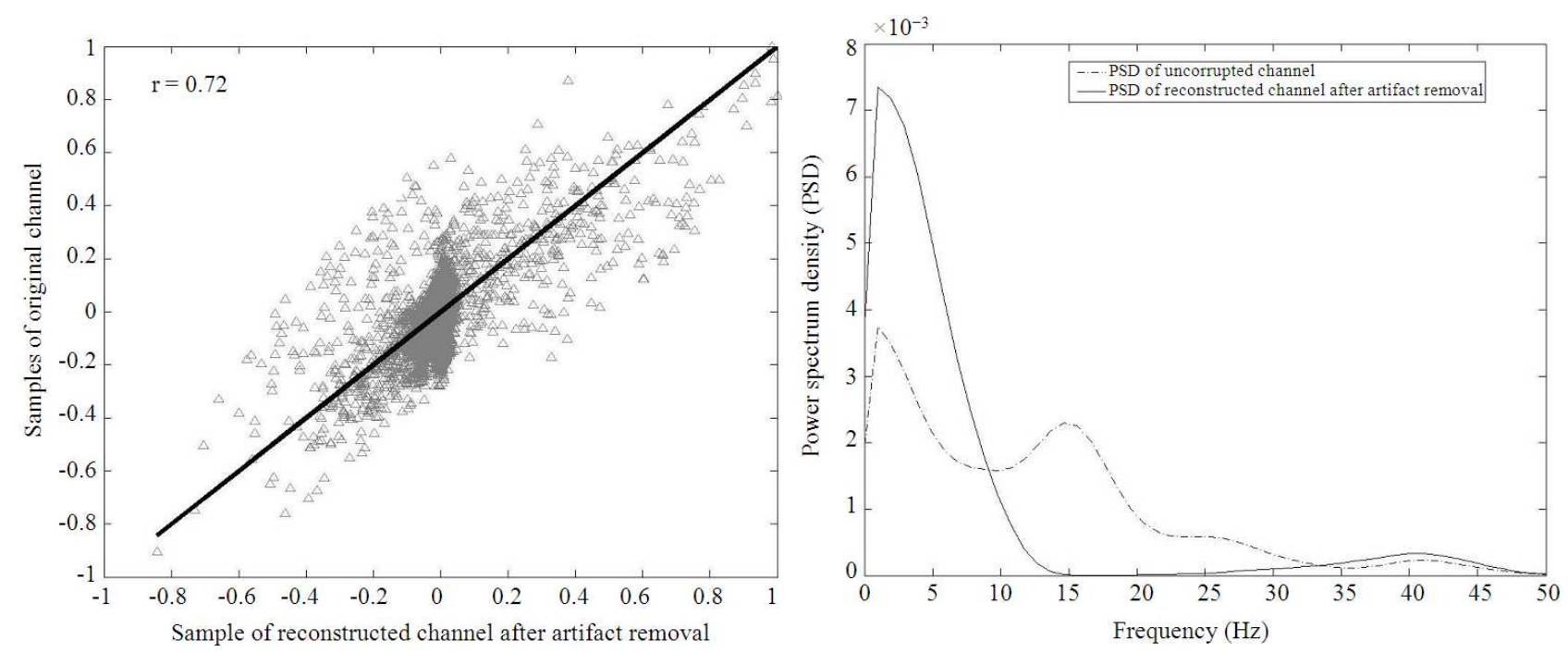

Fig. 5b. Performances of DWT method applied on the corrupted signals by the different artifact typologies shown in Fig. 3. On the top: Comparison on the time- and frequency-domain between the uncorrupted EMG signal and the reconstructed one after impulsive artifact cancellation. The linear regression coefficient is very near to $1(r=0.98)$ with low data dispersion and there is a good agreement between their spectra. On the bottom: comparison on the time- and frequency domain between the uncorrupted EMG signal and the reconstructed one after similar ECG artifact cancellation. The linear regression coefficient is far from $1(\mathrm{r}=0.72)$ with high data dispersion and there are meaningful differences in the frequency range of the spectra in which the artifact is localized

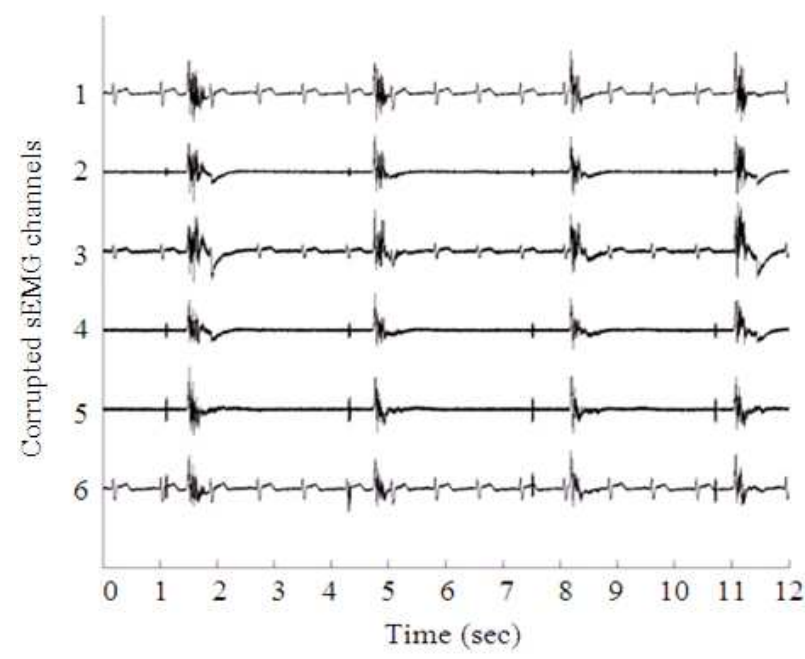

Fig. 6. Synthesized sEMG channels corrupted by impulsiveECG artifact

Comparing the uncorrupted signal with the reconstructed one after the artifact removal, we note that this algorithm works very well with the first typology of artifacts, whereas it is enable to completely remove the second typology of artifacts.

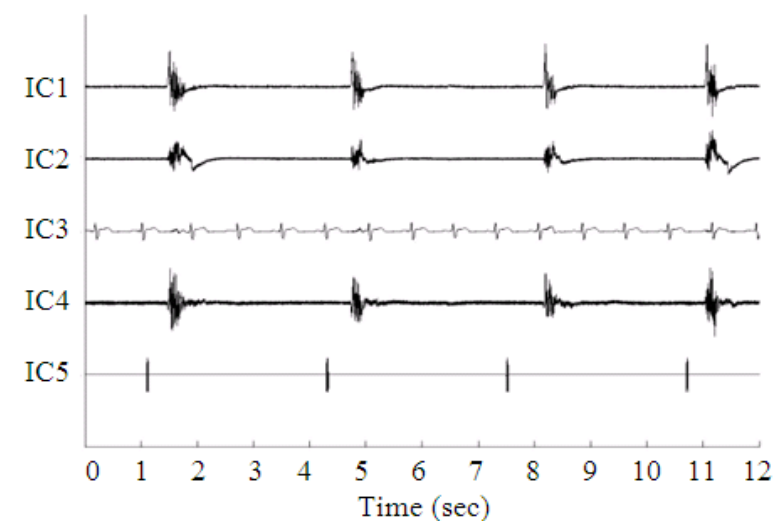

Fig. 7. Independent Components after PCA dimensionality data reduction. IC3 and IC5 represent the artifact contributions

\subsection{Artifact Removal using ICA Method}

Let's consider the synthesized sEMG recordings corrupted by impulsive-ECG artifact, as shown in Fig. 6. This artifact is more difficult to deal with. First, its spectrum is not compactly supported, but it is nonzero in almost all the frequency axis. Moreover, its time shape is different for each recording channel Fig. 7. Independent Components after PCA dimensionality data reduction. IC3 and IC5 represent the artifact contributions 
This is the most suitable kind of signal identifiable by the ICA method. We can see that this approach is able to separate the artifact from the recordings. Figure 7 shows that the 3 rd and the 5 th components represent the artifact contributions that corrupt the sEMG. Thus, suppressing these components and reconstructing the cleaned channels, we can remove the artifact. Figure 8 shows the performances of the ICA method for the first channel. The same results are obtained for the other channels.

This simulation confirms that the ICA method has a very good performance when the recordings are redundant. Let's consider the similar case, but now we suppose to have only three recordings (Fig. 9 and 10) shows that ICA algorithm is not able to isolate the artifact contribution. This occurs when there are more ICs than observed mixtures. In fact, the signals in Fig. 9 are three mixtures of four synthesized independent EMG signals (ICA with over complete bases) and it was solved by modified ICA methods. These methods are rather inefficient and much more complicated. We propose to perform artifact cancellation by WICA rather than modified ICA.

\subsection{Artifact Removal using WICA Method}

The WICA approach is an alternative method to perform artifact removal with respect to ICA with over complete bases. In fact we show that WICA allows performing artifact removal also in the case in which we have no redundancy in sEMG recordings.

Let's apply this method to the corrupted sEMG recordings in Fig. 10. For these corrupted signals the

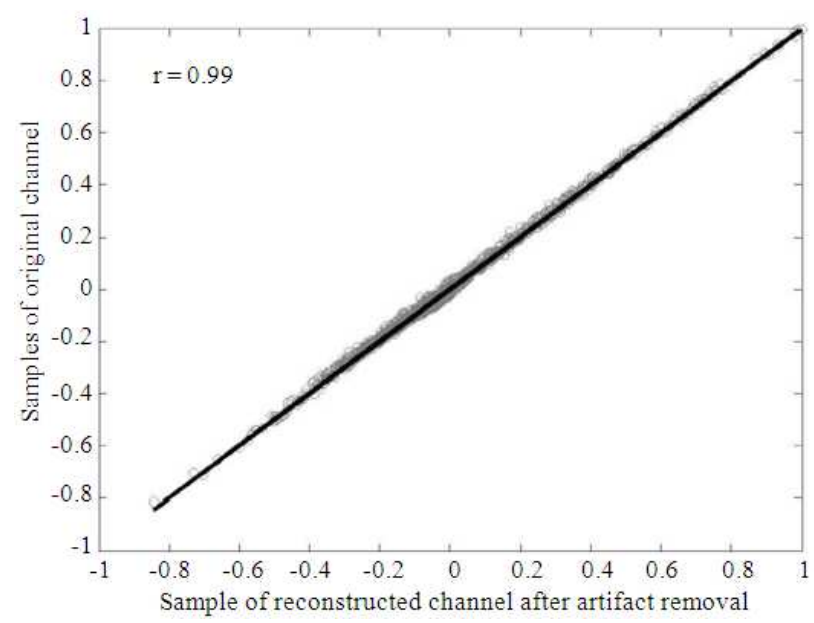

approach has revealed the best ability to separate the original signal by the artifact. Figure $\mathbf{1 1}$ shows the identified artifact by WICA. Figure 12 includes the performance of the proposed method in the time domain and it shows the comparison between the spectra.

Thus, we conclude that the performances of WICA method are better than the ones obtained by the DWT or ICA method. So, the joint use of these techniques allows to perform the artifact cancellation to a wider set of corrupted recordings. In the next section, we will discuss a clinical application in which only the WICA is able to remove the artifacts.

\section{RESULTS: A CLINICAL APPLICATION}

Here, four active electrodes performing a sEMG were put on the pectoral muscles of a healthy subject. We put two electrodes on the right muscle and the other electrode on the left one. In Fig. 13 we show $10 \mathrm{sec}$ of the recordings by sEMG; during the registration session a notch-filter $50 \mathrm{~Hz}$ and a low-pass filter (cutoff frequency $500 \mathrm{~Hz}$ ) were applied; the sampling frequency is $\mathrm{f}_{\mathrm{s}}=1 \mathrm{kHz}$.

Various cycles of simultaneous muscle contraction (both side muscles are contracted synchronously) are recorded by the sEMG. Note that the recordings are highly corrupted by the cardiac artifact. The features of this artifact are comparable with the peculiarities of the synthesized artifact shown in Fig. 9. In order to perform a good analysis of muscle activity, firstly we must remove the artifact corrupting the EMG signals.

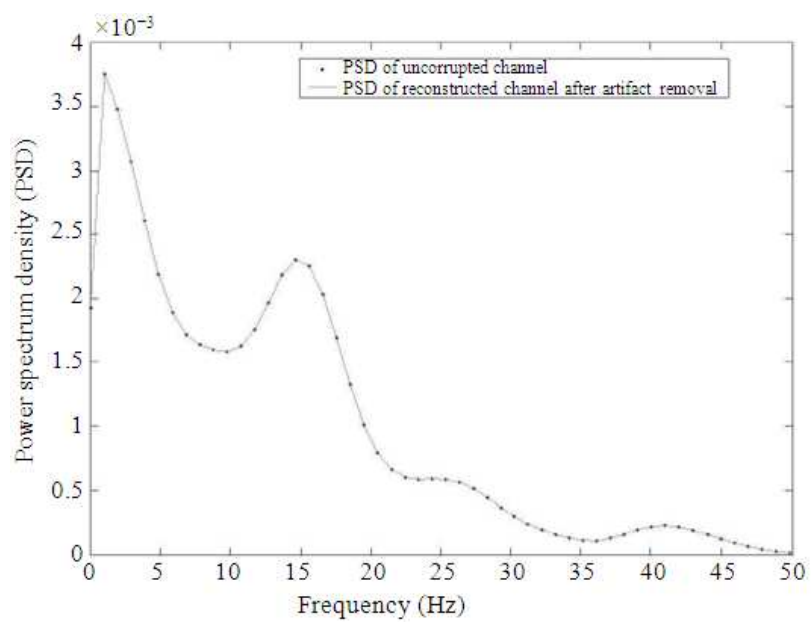

Fig. 8. Testing ICA method. Comparison on the time- and frequency-domain between the uncorrupted first EMG channel and the reconstructed one after impulsive-ECG artifact cancellation. (left) The linear regression coefficient is very near to $1(\mathrm{r}=0.99)$ with low data dispersion and (right) there is a good agreement between their spectra. Similar results are obtained for the other channels 


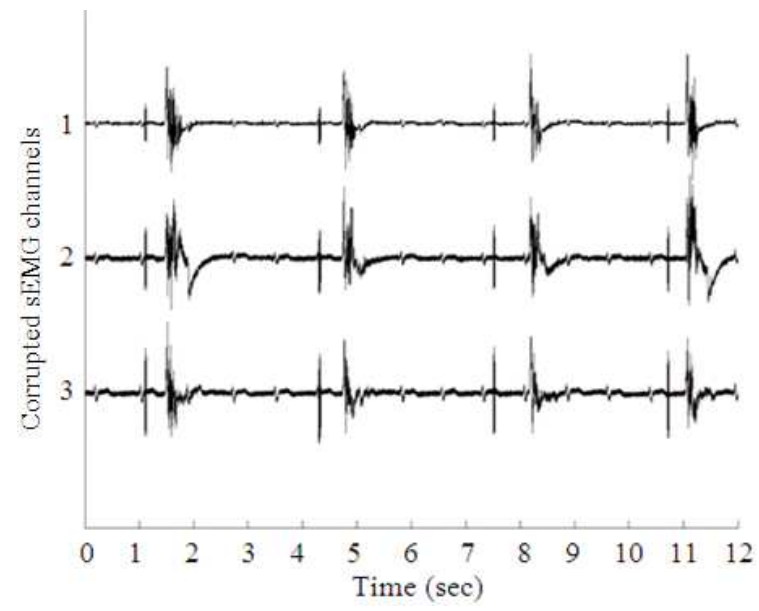

Fig. 9. Three channels of synthesized sEMG signals (channels $1,3,5$ shown on the top of Fig. 2) corrupted by impulsive-ECG artifact

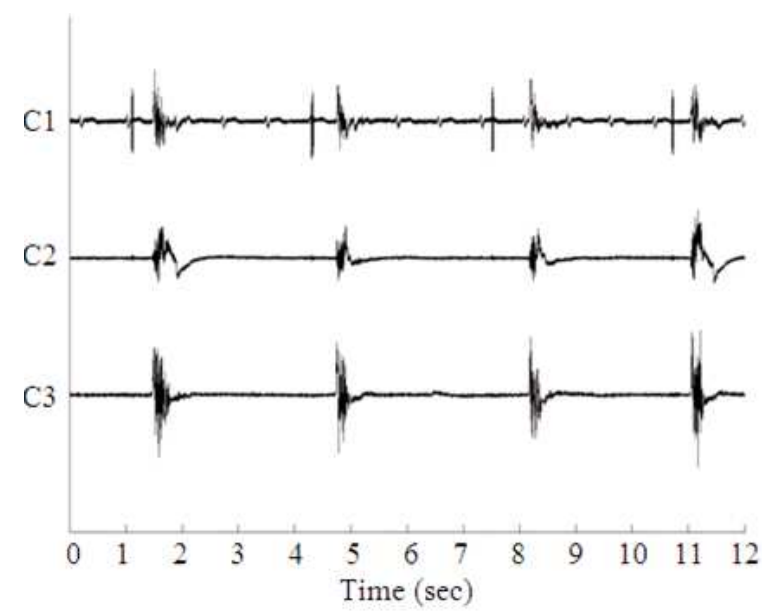

Fig. 10. Independent Components of signals shown in Fig. 9. The ICA algorithm cannot isolate the artifact contributions

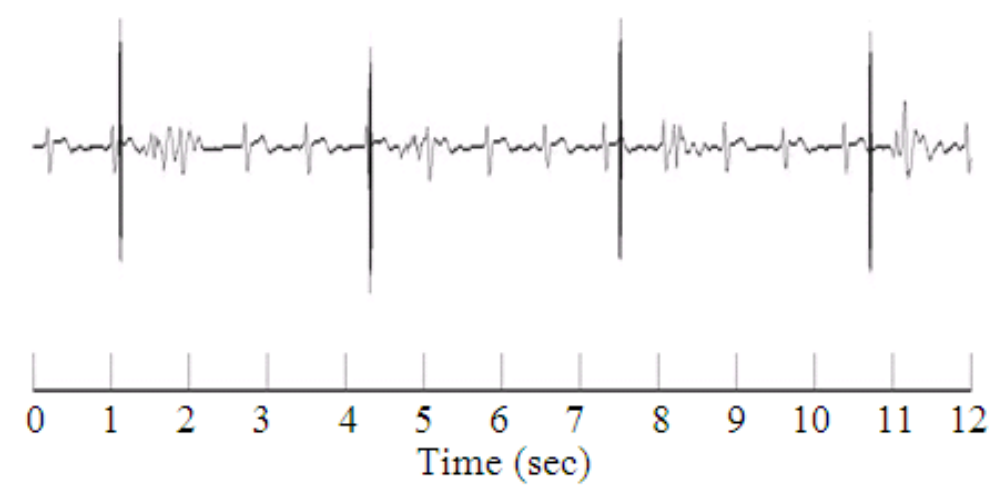

Fig. 11. The artifact identification by the WICA method. The figure shows the identified and removed artifact
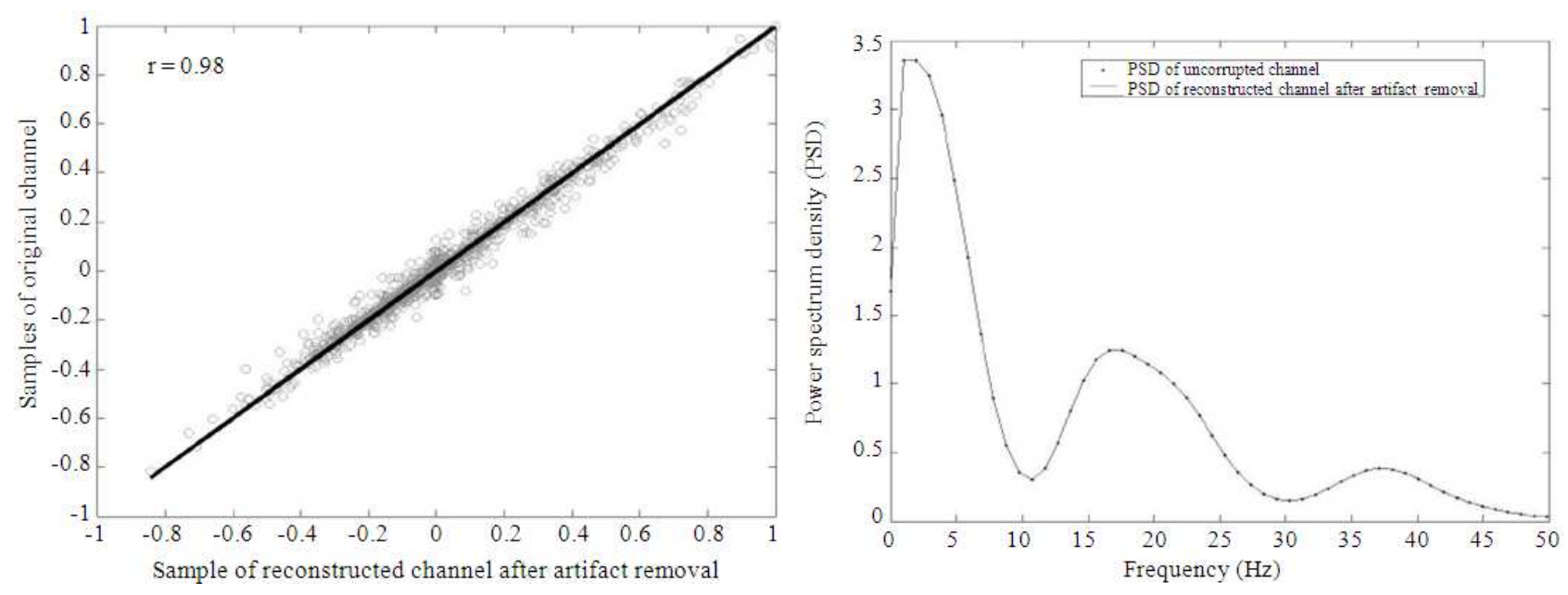
Salvatore Calcagno et al. / American Journal of Applied Sciences 11 (1): 57-68, 2014
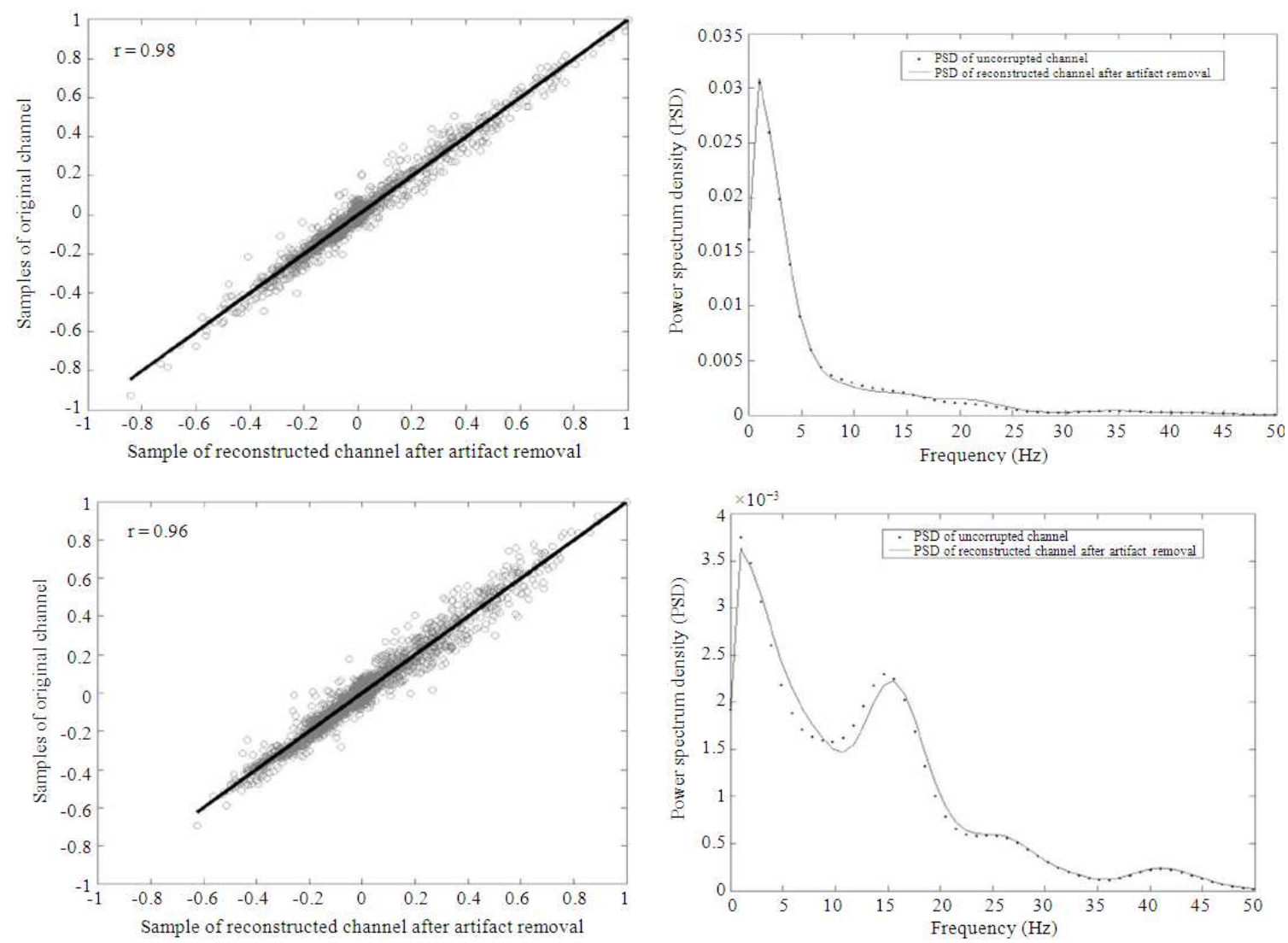

Fig. 12. Testing WICA method. (left) Comparison on the time-domain between the uncorrupted sEMG recordings and the reconstructed ones after impulsive-ECG artifact cancellation. The linear regression coefficients are very near to $1(\mathrm{r}=0.98$ for 1st and 2nd channel and $\mathrm{r}=0.96$ for $3 \mathrm{rd}$ channel) with low data dispersion. (right) Comparison on the frequency-domain between the uncorrupted sEMG recordings and the reconstructed ones after impulsive-ECG artifact cancellation. There is a good agreement between the spectra

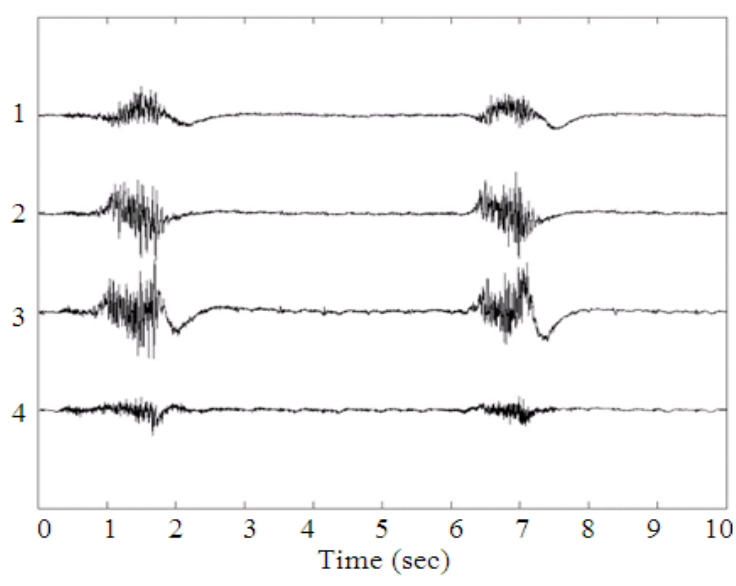

Fig. 13. The pectoral muscles activity recordings performed by sEMG with four electrodes. All the channels are corrupted by a cardiac artifact

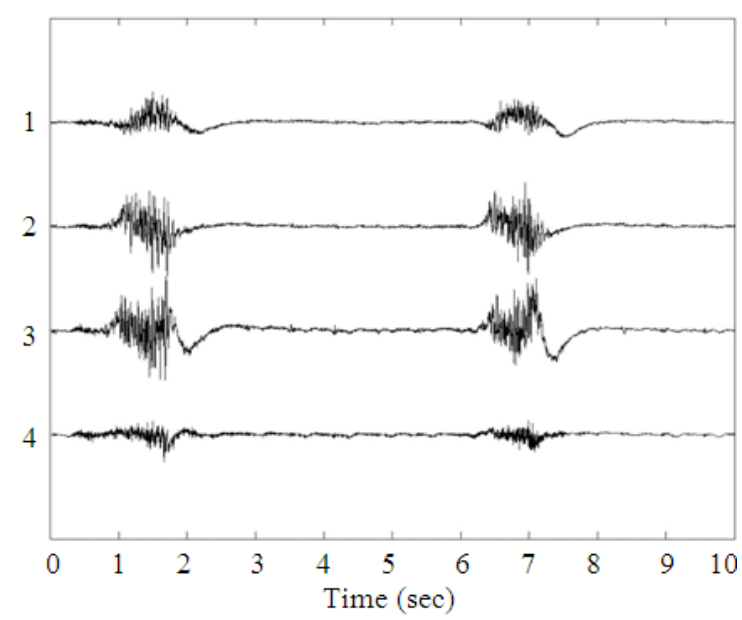

Fig. 14. The reconstructed sEMG recordings after artifact removal by WICA method 


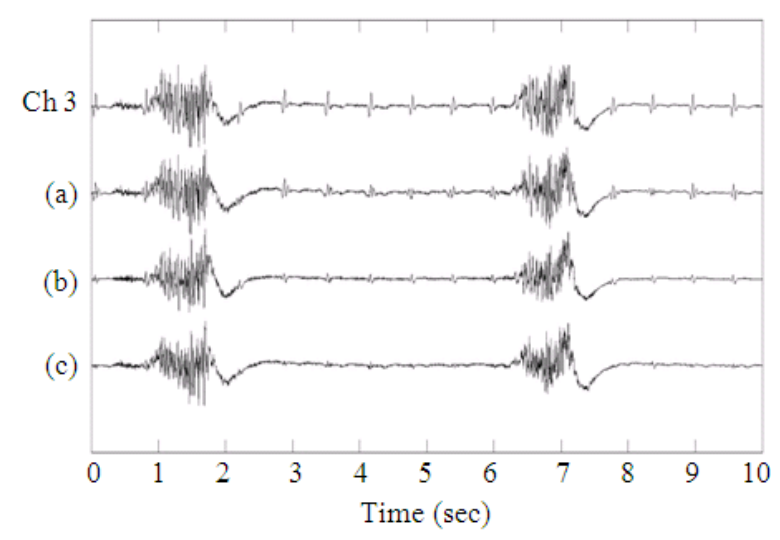

Fig. 15. Comparison among WICA, DWT and ICA method for the most corrupted signal (third channel). WICA reveals $98 \%$ ECG reduction, with respect to the $90 \%$ of the ICA, and to the $73 \%$ of the DWT method

The WICA method allows removing successfully the ECG artifact, as shown in Fig. 14 and $\mathbf{1 5}$ shows the comparison among WICA, DWT and ICA method for the most corrupted electrode (third channel): only our proposed method gives the best results. WICA reveals $98 \%$ ECG reduction, with respect to the $90 \%$ of the ICA and to the $73 \%$ of the DWT method.

\section{CONCLUSION}

In this study, we have presented a comparison among different methods to perform artifact cancellation in biomedical data signal. By synthesized sEMG signals and artifacts, we have compared the performances of the various approaches showing that each approach is suitable for an appropriate kind of artifact. All the presented approaches are based on two algorithms: DWT and ICA. The first is a good method to separate signals which have non-overlapped spectra, whereas the latter is an optimal method to split signals that are statistically independent. We have also studied the performances of a further method, based on a joint application of DWT and ICA, which encompasses the features of both methods. In the final part of the study, the presented algorithms were tested on a real-world signal, which is a sEMG signal from pectoral muscles of a healthy human subject. The results show that the ICA approach allows extending the artifact removal in those clinical applications in which both DWT and ICA, when applied separately, have poor performances. In the future, the proposed technique could will be used together entropy analysis (Morabito et al., 2012; Mammone et al., 2011a; 2011b).

\section{REFERENCES}

Angiulli, G. and M. Versaci, 2002. A neuro-fuzzy network for the design of circular and triangular equilateral microstrip antennas. Int. J. Infrared Millimeter Waves, 23: 1513-1520. DOI: 10.1023/A:1020333704205

Angiulli, G. and M. Versaci, 2003. Resonant frequency evaluation of microstrip antennas using a neuralfuzzy approach. IEEE Trans. Magnet., 39: 1333-1336. DOI: 10.1109/TMAG.2003.810172

Azzerboni, B., G. Finocchio, M. Ipsale, F. La Foresta and M.J. McKeown et al., 2002. Spatio-temporal analysis of surface electromyography signals by independent component and time-scale analysis. Proceedings of the 2nd Joint Engineering in Medicine and Biology, Oct. 23-26, IEEE Xplore Press, pp: 112-113. DOI: 10.1109/IEMBS.2002.1134411

Cacciola, M., F. La Foresta, F.C. Morabito and M. Versaci, 2007. Advanced use of soft computing and eddy current test to evaluate mechanical integrity of metallic plates. NDT E Int., 40: 357-362. DOI: 10.1016/j.ndteint.2006.12.011.

Cacciola, M., G. Megali, S. Calcagno, F.C. Morabito and D. Pellicano et al., 2009. FEA design and misfit minimization for in-depth flaw characterization in metallic plates with eddy current nondestructive testing. IEEE Trans. Magnet., 45: 1506-1509. DOI: 10.1109/TMAG.2009.2012691

Cacciola, M., S. Calcagno, G. Megali, D. Pellicano and M. Versaci et al., 2010. Wavelet coherence and fuzzy subtractive clustering for defect classification in aeronautic CFRP. Proceedings of the International Conference on Complex, Intelligent and Software Intensive Systems, Feb. 15-18, IEEE Xplore Press, Krakow, pp: 101-107. DOI: 10.1109/CISIS.2010.234

Campolo, M., D. Labate, F. La Foresta, F.C. Morabito and A. Lay-Ekuakille et al., 2011. ECG-derived respiratory signal using empirical mode decomposition. Proceedings of the IEEE International Workshop on Medical Measurements and Applications, May 30-31, IEEE Xplore Press, Bari, pp: 399-403. DOI: 10.1109/MeMeA.2011.5966727

Costantino, D., F.C. Morabito, F. Pratico and M. Versaci, 2012. Dynamical reconstruction of road longitudinal profiles: A theoretical and experimental study. Int. J. Mod. Simulat., 32: 149-156. DOI: 10.2316/Journal.205.2012.3.205-4752 
Inuso, G., F. La Foresta, N. Mammone and F.C. Morabito, 2007a. Brain activity investigation by EEG processing: Wavelet analysis, kurtosis and Renyi's entropy for artifact detection. Proceedings of the International Conference on Information Acquisition, Jul. 8-11, IEEE Xplore Press, Seogwipo-si, pp: 195-200. DOI: 10.1109/ICIA.2007.4295725

Inuso, G., F. La Foresta, N. Mammone and F.C. Morabito, 2007b. Wavelet-ICA methodology for efficient artifact removal from Electroencephalographic recordings. Proceedings of the International Joint Conference on Neural Networks, Aug. 12-17, IEEE Xplore Press, Orlando, FL., $\quad$ pp: 1524-1529. DOI: 10.1109/IJCNN.2007.4371184

La Foresta, F., F.C. Morabito, B. Azzerboni and M. Ipsale, 2005. PCA and ICA for the extraction of EEG components in cerebral death assessment. Proceedings of the IEEE International Joint Conference on Neural Networks, Jul. 31-Aug. 4, IEEE Xplore Press, Montreal, Que., pp: 2532-2537. DOI: 10.1109/IJCNN.2005.1556301

Labate, D., F. La Foresta, G. Inuso and F.C. Morabito, 2011a. Multiscale entropy analysis of artifactual EEG recordings. Frontiers Artif. Intell. Applic., 234: 170-177. DOI: 10.3233/978-1- 60750-972-1-170
Labate, D., F. La Foresta, G. Inuso and F.C. Morabito, 2011b. Remarks about wavelet analysis in the EEG artifacts detection. Frontiers Artif. Intell. Applic., 226: 99-106.

Mammone, N., F. La Foresta and F.C. Morabito, 2011b. Discovering network phenomena in the epileptic electroencephalograph through permutation entropy mapping. Front. Artif. Intell. Applic., 226: 260-269.

Mammone, N., F. La Foresta and F.C. Morabito, 2012. Automatic artifact rejection from multichannel scalp EEG by wavelet ICA. IEEE Sensors J., 12: 533-542. DOI: 10.1109/ JSEN.2011.2115236

Mammone, N., G. Inuso, F. La Foresta, M. Versaci and F.C. Morabito, 2011a. Clustering of entropy topography in epileptic electroencephalography. Neural Comput. Applic., 20: 825-833. DOI: 10.1007/s00521-010-0505-2

Morabito, F.C., D. Labate, F. La Foresta, A. Bramanti and G. Morabito et al., 2012. Multivariate multiscale permutation entropy for complexity analysis of Alzheimer's disease EEG. Entropy, 14: 1186-1202. DOI: $10.3390 / \mathrm{e} 14071186$ 